Advancements in Instrumentation

\title{
High Magnification Microscopic System Focusing on Transparent Liquid/Liquid Interface Formed in Thin-Layer Two-Phase Microcell
}

\author{
Satoshi Tsukahara, ${ }^{\dagger}$ Michinori Suehara, and Terufumi FujIwara \\ Department of Chemistry, Graduate School of Science, Hiroshima University, \\ Kagamiyama, Higashi-Hiroshima, Hiroshima 739-8526, Japan
}

\begin{abstract}
A thin-layer two-phase microcell was fabricated without an adhesive, which showed no fluorescence and resisted acids. The lower hollow of the microcell was thin $(0.18 \mathrm{~mm})$, which was in the range of the working distance of the used objective of high magnification (60×) and high numerical aperture (1.2). A reflection method using probe light was newly employed for focusing the objective on a transparent dodecane/water interface strictly with an inverted microscope. The system was applied to in situ fluorescence microscopic measurements of the dynamics of single DNA molecules (165600 base pairs) at the dodecane/water interface.
\end{abstract}

(Received October 16, 2006; Accepted December 18, 2006; Published March 10, 2007)

\section{Introduction}

Liquid/liquid interfaces are extensively investigated by various macroscopic optical methods or thermodynamic methods, because they show specific functions in colloidal chemistry and biochemistry as well as solvent extraction chemistry. ${ }^{1}$ Liquid/liquid interfaces are flexible, and they can not be fixed at a spatial position; these restrictions depress the development of studies on liquid/liquid interfaces. Especially, microscopic investigations on liquid/liquid interfaces have been scarcely reported.

We conducted several studies on in situ microscopic measurements of liquid/liquid interfacial phenomena, ${ }^{1-4}$ and found some specific interfacial inhomogeneities. For example, the microdomain formation of a porphyrin ${ }^{2}$ and the microextraction of $\mathrm{Eu}(\mathrm{III})$ with an extractant ${ }^{3}$ were observed at the toluene/water interface and at the dodecane/water interface, respectively. In these studies, there were several difficulties about microscopic measurements for transparent liquid/liquid interfaces. One of the serious problems was a difficulty in focusing an objective just on the interfaces. An approximate level of the interfaces has been known from some indirect information so far.

An objective of high magnification and high NA (numerical aperture) is favorable to high spatial resolution, but its working distance and focal depth tend to be short. Therefore, for the sake of microscopic measurements of liquid/liquid interfacial phenomena with high spatial resolution, a new method for focusing on the transparent interfaces strictly and a new cell matching the short working distance are essential.

Deoxyribonucleic acid (DNA) is one of the important key substances in biology. It has been found by fluorescence microscopy that single DNA molecules show several kinds of motion in aqueous solutions, such as conformational change, intramolecular motion and three-dimensional Brownian

$\dagger$ To whom correspondence should be addressed.

E-mail: tsuka@sci.hiroshima-u.ac.jp motion..$^{5-7}$ Furthermore, it is known that the conformational change or apparent size of DNA molecules is deeply related to their hydration. So far, the dynamics of single DNA molecules has been examined in homogeneous aqueous solutions, but it has been scarcely studied at interfaces, where the physical property changes drastically.

Several macroscopic studies have included the behaviors of DNA in two-phase systems. For example, the solvent extraction of DNA by cationic surfactants was reported, ${ }^{8}$ in which DNA passed through the liquid/liquid interface. Furthermore, liquid/liquid interfaces are sometimes treated as a model of biological membranes. Therefore, the dynamic behavior of single DNA molecules at bare liquid/liquid interfaces is a basic and important research subject.

In the present study, a non-fluorescent cell is fabricated, which contains dodecane and a DNA aqueous solution and which matches the short working distance of the objective. Next, a reflection method is newly employed for focusing an objective of high magnification and high NA on a transparent liquid/liquid interface with an inverted fluorescence microscope. The dynamic behaviors of single DNA molecules are measured at the dodecane/water interface with the constructed system.

\section{Experimental}

\section{Reagents and materials}

Giant double-strand DNA (T4GT7DNA, 165600 base pairs) was purchased from Nippon Gene, which was labeled with a fluorescent dye, DAPI (4',6-diamidino-2-phenylindole, Aldrich). Water was purified with a Milli-Q system (Milli-Q SP, Millipore). Other reagents were of analytical reagent grade. A DNA aqueous solution contained $2.0 \times 10^{-12} \mathrm{M}(1 \mathrm{M}=1$ mol $\mathrm{dm}^{-3}$ ) DNA, $3.9 \times 10^{-7} \mathrm{M}$ DAPI, $0.010 \mathrm{M}$ tris(hydroxymethyl)aminoethane, and $1.0 \times 10^{-3} \mathrm{M}$ EDTA; the $\mathrm{pH}$ of the DNA solution was 8.0. For reducing the photobleach of DAPI, 2-mercaptoethanol was added to the DNA solution by $4 \%(\mathrm{v} / \mathrm{v})$ before experiments. 


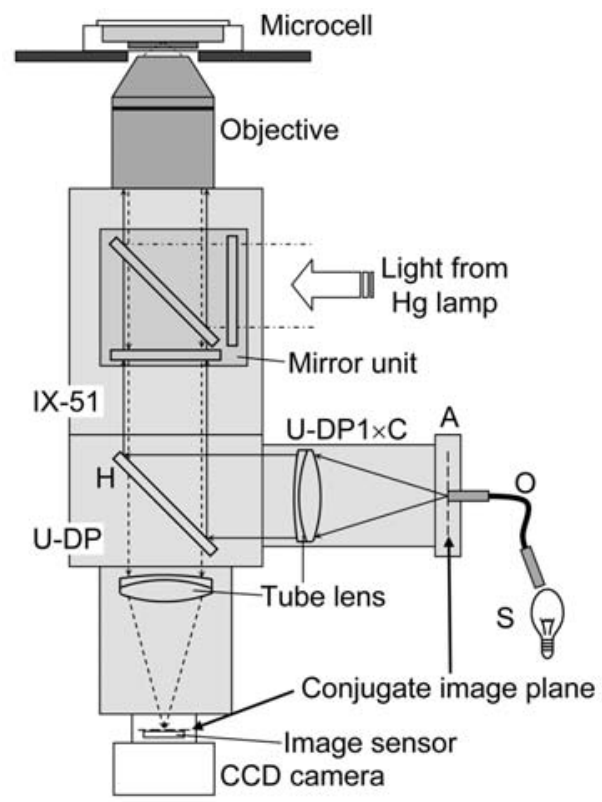

Fig. 1 Schematic illustration of the inverted fluorescence microscope and the optical system for the reflection method. Abbreviations: A, home-made C-mount adaptor; O, optical fiber; $\mathrm{S}$, light source; $\mathrm{H}$, half-mirror.

Two membrane filters $(0.2$ and $0.02 \mu \mathrm{m}$ in pore size, Advantec and Whatman, respectively) were used for removing a few tens nm-level dust particles in solvents and solutions.

\section{Microscopic system}

The fluorescence microscope used in the present study is schematically shown in Fig. 1. It consists of a common inverted microscope (IX-51, Olympus), an objective (UPlanApo, 60×, NA 1.2, water-immersion, working distance $0.25 \mathrm{~mm}$, Olympus), a mirror unit (U-MWU2, Olympus), and a light source for fluorescence $(\mathrm{Hg}$ discharge lamp $(100 \mathrm{~W}))$. The mirror unit contains an excitation filter, a dichromatic mirror, and an emission filter; it can set the excitation and observation wavelengths at $330-385 \mathrm{~nm}$ and $430-900 \mathrm{~nm}$, respectively. A dual port unit (U-DP, Olympus) with a half-mirror, its C-mount unit (U-DP1 $\times$ C, Olympus) including a tube lens, a home-made C-mount adaptor, and an optical fiber with FC-type connectors (300 $\mu \mathrm{m}$ in diameter, NA 0.2, Hamamatsu Photonics) were used for introducing white light from another light source (a tungsten halogen lamp, $100 \mathrm{~W}$ ) to the microscope.

Microscopic pictures were obtained with a monochromatic CCD camera (WAT-100N, Watec) at the video rate (33 $\mathrm{ms} /$ frame). The pictures were recorded on a video tape, and were then transferred to a personal computer as digital pictures for analysis. All of the experiments were carried out at 23 $27^{\circ} \mathrm{C}$.

\section{Results and Discussion}

Fabrication of non-fluorescent thin-layer two-phase microcell

As for in situ fluorescence microscopic measurements of the dynamics of single macromolecules, such a DNA in solutions, the following three requirements should be satisfied: 1) a cell of no fluorescence, 2) high magnification and high spatial resolution, and 3) no dust particles in solution.

In the previous work, a thin-layer two-phase microcell was
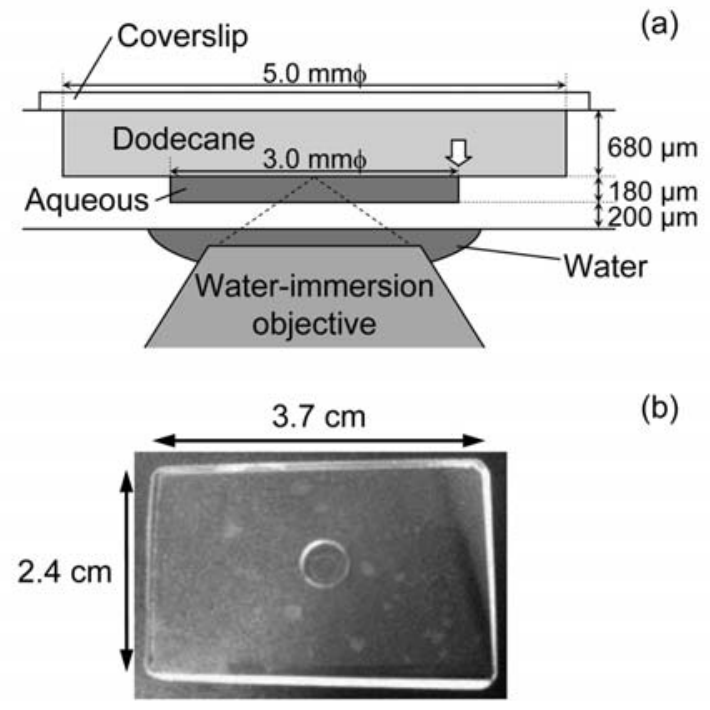

(b)

Fig. 2 (a) Schematic illustration of the fabricated thin-layer twophase microcell, in which the dodecane/water interface was prepared, and (b) photograph of the microcell.

developed for the sake of in situ microscopic measurements of molecular diffusion at liquid/liquid interfaces, ${ }^{4}$ whose structure was similar to that shown in Fig. 2. It was fabricated with three pieces of glass plate and epoxy adhesive. Although the epoxy adhesive resists water and almost all of organic solvents, it shows unfavorable fluorescence. Furthermore, the microcell can not be washed with concentrated nitric acid, because epoxy adhesive does not resist acids. Thus, the microcell with epoxy adhesive satisfies only the above second requirement.

For satisfying all of the three requirements, a thin-layer twophase microcell should be fabricated without an epoxy adhesive. It is known that glass plates of flat and fine surfaces adhere to each other by heat and pressure. In the present study, this technique was employed for fabricating the microcell. First, surfaces of a non-bored and a bored $(3.0 \mathrm{~mm}$ in diameter) borosilicate glass plates were polished precisely. Then, they were pressed with carbon weights $(0.5 \mathrm{~kg})$ in an oven. The oven temperature was raised from room temperature to $700^{\circ} \mathrm{C}$ (below the glass transition temperature by $120^{\circ} \mathrm{C}$ ) for $1 \mathrm{~h}$, and kept at $700^{\circ} \mathrm{C}$ for $10 \mathrm{~min}$, and then lowered to room temperature in $3 \mathrm{~h}$. Next, the upper surface was polished again, which then adhered to another bored $(5.0 \mathrm{~mm}$ in diameter) borosilicate glass plate by the same technique. Finally, the top and bottom surfaces were polished. The final size and a photograph of the fabricated microcell are shown in Figs. 2(a) and (b), respectively. The thickness of the lower hollow is shorter than the working distance of the objective $(0.25 \mathrm{~mm}),{ }^{9}$ and the microcell shows no fluorescence and resists acids.

\section{Preparation of clean dodecane/water interface}

The fabricated microcell was washed with acetone and water, sunk in concentrated nitric acid for $2-3 \mathrm{~h}$, washed with water many times and dried with pure $\mathrm{N}_{2}$ gas just before use. This procedure effectively removed any dust particles from the inner wall of the microcell.

There were many dust particles in the purchased dodecane and the DNA-free aqueous solutions. Distillation could not remove dust particles in dodecane, probably because dust particles in air were incorporated in the solvent again. On the other hand, it was found that the filtration with the two membrane filters $(0.2$ 

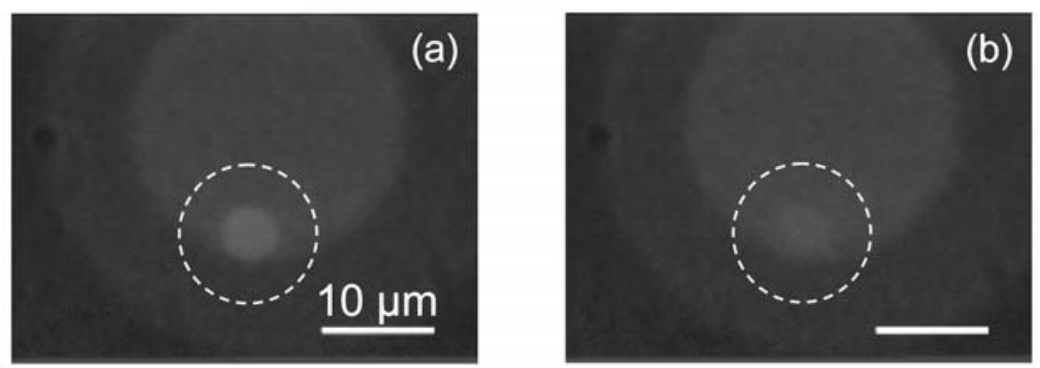

Fig. 3 Focusing on the transparent dodecane/water interface. The bright spots in the circles were the images of the fiber exit, which were formed with the probe light reflected at the interface. The interface was (a) in focus and (b) out of focus (at $4 \mu \mathrm{m}$ below the interface).

and $0.02 \mu \mathrm{m}$ in pore size) successfully removed the fine dust particles in dodecane and the aqueous solutions.

Dodecane, the DNA-free aqueous solutions, and 2mercaptoethanol were filtrated with the 0.2 and $0.02 \mu \mathrm{m}$ membrane filters in this sequence just before use. The aqueous solutions were mixed with the purchased DNA solution and the 2-mercaptoethanol. Next, an aliquot of the resultant aqueous DNA solution was put into the lower hollow of the microcell. Then, an aliquot of the dodecane was added on the aqueous solution quietly, and the dodecane phase was covered with a cut coverslip $(10 \times 18 \mathrm{~mm})$ to close the microcell.

\section{Focusing on transparent dodecane/water interface}

Different from solid/liquid and solid/air interfaces, transparent liquid/liquid interfaces have no cracks. Thus, it is impossible to focus on the liquid/liquid interfaces without a probe. Since the horizontal level of the microcell edge, indicated by the white axis in Fig. 2(a), almost agrees with that of the liquid/liquid interface, the microcell is convenient for focusing on a transparent liquid/liquid interface approximately. On the other hand, in the case of an objective of high NA, the focal depth is short, and thus the level of the interface must be determined strictly. For this purpose, the following method using probe light is newly applied.

In the case of the dodecane/water system, light is reflected at the interface and it is not reflected in bulk solutions. Commonly, the reflectance $(R)$ at a transparent interface between phases 1 and 2 depends on the difference in the refractive index $(n)$ of the phases and the incident angle. In the case of irradiation normal to the interface, $R$ is expressed as: ${ }^{10}$

$$
R=\left(\frac{n_{1}-n_{2}}{n_{1}+n_{2}}\right)^{2},
$$

where the subscripts 1 and 2 refer to the phases 1 and 2, respectively. The $R$ values are estimated to be 0.0044 and 0.0011 at the glass/water and the dodecane/water interfaces, respectively.

One exit of the optical fiber is located at the conjugate image plane of a specimen for the U-DP1 $\times$ C unit, as shown in Fig. 1 . White light is introduced from the other exit of the optical fiber as probe light. The probe light is collimated by the tube lens and reflected by the half-mirror, and then strikes the specimen through the objective. Therefore, when a reflective specimen is in focus, a focused image of the fiber exit is formed on the specimen. A fraction of the probe light reflected on the specimen is collected by the same objective and passes through the half-mirror. The CCD image sensor is also located at the conjugate image plane of the specimen, which means that the focused images of the specimen and the fiber exit are superimposed on the CCD image sensor.

The microcell including dodecane and water in it has five interfaces, as shown in Fig. 2(a): two glass/water interfaces, a dodecane/water interface, a glass/dodecane interface, and an air/glass interface, from the bottom. In this case, only the two glass/water interfaces and the dodecane/water interface are in the range of the working distance of the objective. When the focal plane was moved up from the bottom of the microcell, the image of the fiber exit appeared three times; the first and second images were bright and settled, but the third one was dark and it slightly fluctuated when the microscope stage was hit gently. Therefore, the third one corresponds to the dodecane/water interface.

The diameter of the fiber exit and the magnification of the objective were $300 \mu \mathrm{m}$ and $60 \times$, respectively, and thus an image of the fiber exit of $5 \mu \mathrm{m}(=300 \mu \mathrm{m} / 60)$ in diameter was observed on the dodecane/water interface. When the interface was in focus, a focused image of the fiber exit appeared, as shown in Fig. 3(a). On the other hand, when the interface was out of focus, the image was blurred, as shown in Fig. 3(b). The accuracy of this method was found to be smaller than $1 \mu \mathrm{m}$. The focal depth $\left(d_{\mathrm{f}}\right)$ is expressed as: $d_{\mathrm{f}}=\lambda / \mathrm{NA}^{2}$, where $\lambda$ is the wavelength of the observed light. With $500 \mathrm{~nm}$ light, $d_{\mathrm{f}}$ is estimated to be $0.35 \mu \mathrm{m}$, which approximately agrees with the accuracy.

As mentioned above, the probe light is reflected on the halfmirror first, and thus the figure tolerance of the mirror is important for sharp imaging of the fiber exit. In the present study, a half-mirror of $2.5 \lambda$ ( $\lambda$ is $632 \mathrm{~nm})$ in figure tolerance was used.

\section{Dynamics of single DNA molecules near the dodecane/water interface}

The lateral resolution of optical microscopy $(d)$ is controlled by the diffraction limit of light, which is expressed as: $d=$ $\lambda /(2 \mathrm{NA})$. For a high spatial resolution, a short wavelength and a high NA are needed. In the present study, DAPI was selected because it emits a fluorescence of short wavelength $(460 \mathrm{~nm})$. The $d$ value for the fluorescence of DAPI was estimated to be $190 \mathrm{~nm}$.

If the T4GT7DNA is fully stretched, its length and radius are $57 \mu \mathrm{m}$ and $1 \mathrm{~nm}$, respectively. In aqueous solutions, DNA molecules show fast intramolecular motions at room temperature, and they are round like balls of wool. When the T4GT7DNA is fully hydrated, that is, in the random-coil state, its observed size is $2-4 \mu \mathrm{m}$ by optical microscopy. ${ }^{5}$

The number of DNA molecules being in focus was counted in the field of view $(42 \times 31 \mu \mathrm{m})$ of the fluorescence microscope 
(a)

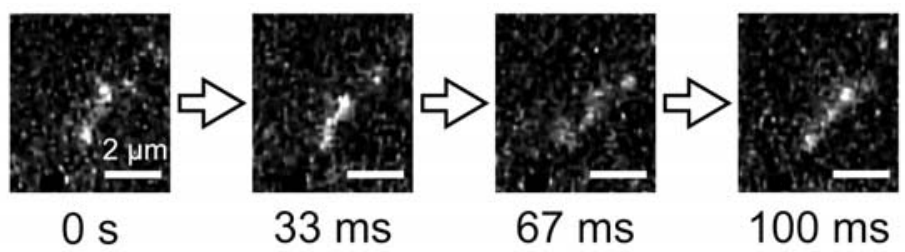

(b)

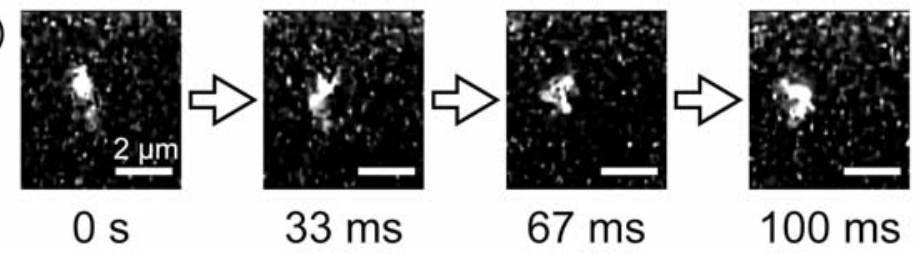

Fig. 4 Continuous fluorescence microscopic pictures of one single DNA molecule (a) in the aqueous phase and (b) near the dodecane/water interface. The contrast was adjusted for clear images.

by changing the focal plane. The hydrophilic and poly-anionic DNA molecules did not exist in the dodecane phase. In the aqueous phase, many single DNA molecules were found, and they were distributed homogeneously; they showed a threedimensional Brownian motion and a fast intramolecular motion, as shown in Fig. 4(a). The observed size of the DNA molecules was in the range of $2-3 \mu \mathrm{m}$, which agreed well with the reported value. ${ }^{5}$ On the other hand, the number of DNA molecules near the bare dodecane/water interface was as many as that in the aqueous phase, and they showed a threedimensional Brownian motion, which indicated that the DNA molecules were hardly adsorbed at the interface.

A fast intramolecular motion was also observed for DNA molecules near the dodecane/water interface, as shown in Fig. 4(b). The size of the DNA molecules near the interface was in a range of $1-2 \mu \mathrm{m}$, which tended to be smaller than that in the aqueous phase. It is known that DNA molecules shrink when the degree of their hydration is lowered. The DNA molecules were not adsorbed at the dodecane/water interface, but their hydration might have been weakened as they approached the interface where the activity of water could be lowered. Further investigations should be done for the dynamic behaviors of single DNA molecules at the liquid/liquid interface as well as DNA hydration there.

\section{Conclusions}

The dynamics of single DNA molecules near the dodecane/water interface was measured with a fluorescence microscope and an objective of high magnification $(60 \times)$ and high NA (1.2). The dodecane/water interface was prepared in a non-fluorescent thin-layer two-phase microcell. The DNA molecules tended to shrink at the interface compared with those in the aqueous phase, which might have been caused by the difference in their hydration. In the future, the dynamics of single DNA molecules should be investigated at liquid/liquid interfaces in the presence of some surfactants or proteins.
The reflection method was newly introduced, which enabled one to focus on the transparent dodecane/water interface. This method will be applied to other microscopic studies on liquid/liquid interfaces.

\section{Acknowledgements}

The authors are grateful to the glassblowing section in the Technical Center at Hiroshima University for fabricating the thin-layer two-phase microcell. This work was supported by a Grant-in-Aid for Scientific Research (B) from the Ministry of Education, Science, Sports and Culture, Japan (No. 16350046).

\section{References and Footnotes}

1. S. Tsukahara, Anal. Chim. Acta, 2006, 556, 16.

2. N. Fujiwara, S. Tsukahara, and H. Watarai, Langmuir, 2001, 17, 5337.

3. S. Tsukahara, A. Takata, and H. Watarai, Anal. Sci., 2004, 20, 1515 .

4. F. Hashimoto, S. Tsukahara, and H. Watarai, Langmuir, 2003, 19, 4197.

5. K. Yoshikawa and Y. Matsuzawa, Physica D, 1995, 84, 220.

6. R. S. Dias, J. Innerlohinger, O. Glatter, M. G. Miguel, and B. Lindman, J. Phys. Chem., 2005, 109, 10463.

7. S. M. Mel'nikov, V. G. Sergeyev, and K. Yoshikawa, J. Am. Chem. Soc., 1995, 117, 2401.

8. M. Goto, A. Momota, and T. Ono, J. Chem. Eng. Jpn., 2004, 37, 662 .

9. The used objective is one of the objectives suitable for biological purposes, whose given working distance does not include the thickness of coverslip.

10. W. N. Hansen, in "Advances in Electrochemisty and Electrochemical Engineering”, ed. C. W. Tobias, 1973, Vol. 9, John Wiley and Sons, New York, 1 - 60. 